\title{
Polymer drag reduction in exact coherent structures of plane shear flow
}

\author{
Philip A. Stone \\ Department of Chemical and Biological Engineering, University of Wisconsin-Madison, Madison, \\ Wisconsin 53706-1607 \\ Anshuman Roy and Ronald G. Larson \\ Department of Chemical Engineering, University of Michigan, Ann Arbor, Michigan 48109-2136 \\ Fabian Waleffe \\ Departments of Mathematics and Engineering Physics, University of Wisconsin-Madison, Madison, \\ Wisconsin 53706-1388 \\ Michael D. Graham ${ }^{\mathrm{a})}$ \\ Department of Chemical and Biological Engineering, University of Wisconsin-Madison, Madison, \\ Wisconsin 53706-1607
}

(Received 9 March 2004; accepted 2 June 2004; published online 6 August 2004; publisher error corrected 16 September 2004)

\begin{abstract}
Recently discovered traveling-wave solutions to the Navier-Stokes equations in plane shear geometries provide model flows for the study of turbulent drag reduction by polymer additives. These solutions, or "exact coherent states" (ECS), qualitatively capture the dominant structure of the near-wall buffer region of shear turbulence, i.e., counter-rotating pairs of streamwise-aligned vortices flanking a low-speed streak in the streamwise velocity. The optimum length scales for the ECS match well the length scales of the turbulent coherent structures and evidence suggests that the ECS underlie the dynamics of these structures. We study here the effect of viscoelasticity on these states. The changes to the velocity field for the viscoelastic ECS, where the FENE-P model calculates the polymer stress, mirror the modifications seen in experiments of fully turbulent flows of polymer solutions at low to moderate levels of drag reduction: drag is reduced, streamwise velocity fluctuations increase while wall-normal fluctuations decrease, and smaller wavelength structures are suppressed. These modifications to the ECS are due to the suppression of the streamwise vortices. The polymer molecules become highly stretched in the wavy, streamwise streaks, where the flow is predominately elongational, then relax as they move from the streaks into and around the streamwise vortices, where the flow is predominately rotational. This relaxation of the polymer molecules produces a force that directly opposes the fluid motion in the vortices, weakening them. Since the pressure fluctuations have their greatest magnitude (i.e., they are most negative) in the cores of the vortices, a reduction in vortex strength leads to a decrease in the magnitude of the pressure fluctuations. The pressure fluctuations redistribute energy from the streamwise velocity fluctuations to the Reynolds shear stress, so a decrease in their magnitude leads to a reduction in turbulent drag. For the viscoelastic ECS, we also find that after the onset of drag reduction (at Weissenberg number, $\mathrm{We} \approx 7$ ) there is a dramatic increase in the critical wall-normal length scale at which the ECS can exist. This sharp increase in length scale mirrors experimental observations and is also consistent with the observed shift to higher Reynolds numbers of the transition to turbulence in polymer solutions. (C) 2004 American Institute of Physics.
\end{abstract}

[DOI: $10.1063 / 1.1775192]$

\section{INTRODUCTION}

The decrease due to polymer additives of skin friction in turbulent flow has received much attention since the discovery of the phenomenon in the $1940 \mathrm{~s} .{ }^{1-4}$ Very small polymer concentrations, on the order of ten parts per million by weight, can lead to reductions in drag of $50 \%$ or greater. At these small concentrations the properties of the solution measured in simple shear flows do not deviate appreciably from those of the pure solvent. However, in extensional flows,

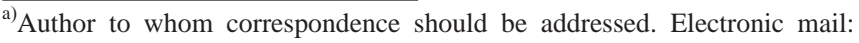
graham@engr.wisc.edu
}

large stresses are generated due to the polymer contribution to the extensional viscosity. It is recognized that the extensional behavior of the polymers is key to the phenomenon of drag reduction, but a detailed knowledge of how polymer stretch and relaxation interact with turbulent structure is not available. The goal of the present work is to better understand this interaction, particularly in the context of near-wall turbulence.

Observations of drag-reducing fluids indicate that, at least near the onset Reynolds number for drag reduction, the effects of the polymer are confined primarily to the near-wall buffer layer, ${ }^{1,5-8}$ which is the most important region for the production and dissipation of turbulent energy. ${ }^{9}$ From experi- 
mental observations and direct numerical simulation (DNS) of turbulent flows, the dominant structures of the buffer region are found to be pairs of counter-rotating, streamwisealigned vortices. ${ }^{10,11}$ These vortices pull slower moving fluid away from the wall, forming low-speed, streamwise velocity streaks. There is a characteristic behavior of these streaks known as "bursting" that produces turbulent kinetic energy in the buffer region. During a "bursting" event, the downstream end of a streak moves rapidly away from the wall. This part of the process, known as "ejection," has positive $v_{y}^{\prime}$ (wall-normal fluctuating velocity) and negative $v_{x}^{\prime}$ (streamwise fluctuating velocity). Continuity demands that the "ejection" be accompanied by a "sweep" that brings fastmoving fluid near the wall (i.e., $v_{y}^{\prime}<0$ and $v_{x}^{\prime}>0$ ). The production of kinetic energy is $-\overline{v_{x}^{\prime} v_{y}^{\prime}} \partial \bar{V} / \partial y$, where $\bar{V}$ is the mean velocity in the $x$-direction (and overbar denotes ensemble average). ${ }^{11}$ Given this equation for production, it is easy to see that the signs of the velocities associated with both "ejections" and "sweeps" give positive kinetic energy production.

In flows exhibiting drag reduction, the structure of the buffer region is modified. Most notably, the wall-normal thickness of the buffer region increases, ${ }^{1}$ the coherent structures in this region shift to larger length scales, ${ }^{5,12-14}$ and the bursting rate decreases. ${ }^{5}$ These structural changes are accompanied by changes in the root-mean-square (rms) velocity fluctuations and Reynolds stresses. Namely, the wall-normal and spanwise fluctuations are reduced while, at least at low to moderate degrees of drag reduction, the streamwise velocity fluctuations are enhanced. ${ }^{15}$ Streamwise vorticity fluctuations are also decreased. ${ }^{12}$ Perhaps most importantly, the Reynolds shear stress decreases. ${ }^{12,16,17}$ These changes become more pronounced as the extensional viscosity of the polymer solution is increased. ${ }^{8,16,18,19}$

Due to the importance of the buffer region structures in the production of turbulent energy and the observation that these structures are modified in drag-reducing flows, they are the focus of the present study. To perform the study in a well-controlled way, we make use of an "exact" model of these structures, the family of so-called "exact coherent states" (ECS) recently found in plane shear flows. ${ }^{20-26}$ The ECS are traveling-wave solutions of the Navier-Stokes equations that capture the dominant buffer region structure, i.e., counter-rotating, streamwise-aligned vortex pairs that flank streaks in the streamwise velocity. ${ }^{9,26,27}$ The structure of an ECS in plane Couette flow is seen in Fig. 1. The isosurfaces of streamwise vorticity clearly show a counter-rotating pair of streamwise vortices, while the contours of streamwise velocity show the high speed (white) and low speed (black) streaks brought about by the vortices. This is the same structure that is seen to dominate the buffer region. Traveling wave solutions of this structure are found in plane Couette and Poiseuille flows using both constant vorticity and no-slip boundary conditions as well as in pipe flow. ${ }^{26,28}$ For the variant of plane Couette flow studied here, which is described in detail in Sec. II, there is a trivial (laminar) solution to the Navier-Stokes equations at all Reynolds numbers, Re. At $\operatorname{Re} \approx 100$, two traveling-wave solutions appear as further de-

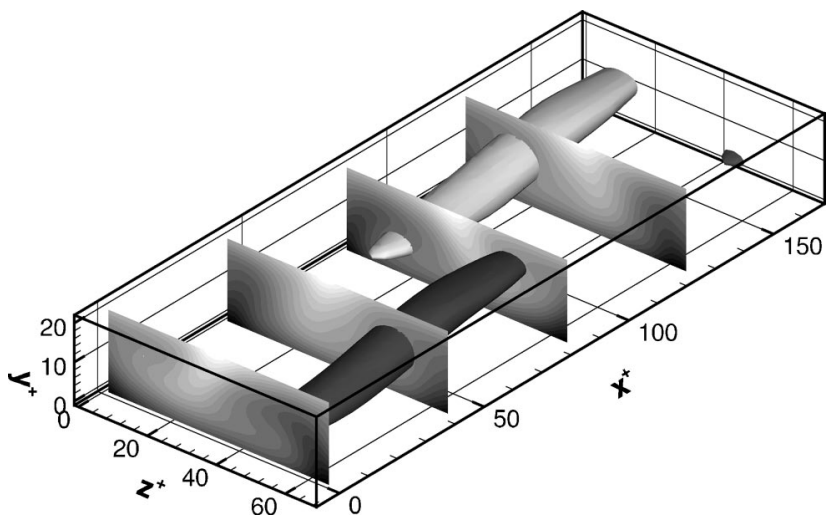

FIG. 1. "Exact coherent state" in plane Couette flow with constant vorticity boundary conditions. "High drag" state at $\mathrm{Re}=110$. The contours are of $v_{x}$ (white is positive, black is negative). The constant vorticity surfaces are $\omega_{x}^{+}=-0.36$ (dark gray) and $\omega_{x}^{+}=0.36$ (light gray)

tailed in Sec. III. The simultaneous appearance of two steady states as a parameter increases, Re in this case, is a saddlenode bifurcation, also referred to as a limit point or fold. ${ }^{29}$ While both of these solutions are unstable, one of these states, which we will call the "high drag" state due to its lower mean velocity at a given Reynolds number, has greater stability relative to the "low drag" ECS-the "high drag" solutions have one more stable direction in phase space than the "low drag" solutions. Experimentally, Bottin et al. have stabilized an ECS-like steady state in a Couette flow with countermoving walls by placing a thin, spanwise-oriented wire in the zero-velocity plane. ${ }^{30}$ The wire provides a finite amplitude disturbance to the flow as well as pinning the location of the flow pattern.

The appearance of the ECS presages the transition to turbulence. For Couette flow with no-slip boundary conditions, the ECS appear at $\operatorname{Re} \approx 128,{ }^{26}$ while persistent turbulence is seen experimentally for $\mathrm{Re} \geq 325{ }^{30}$ In channel flow, the ECS arise at $\operatorname{Re} \approx 650 ;{ }^{26}$ experimentally, the transition to turbulence is at $\operatorname{Re} \approx 1000 .{ }^{31}$ For pipe flow $\operatorname{Re} \approx 1300$ (Ref. 28) for the appearance of the ECS and $\operatorname{Re} \approx 2100$ (Ref. 32) for transition. Note that these experimental values for transition are for the existence of persistent, fully developed turbulence, but intermittent turbulent spots can appear well below these values.

For the ECS in plane Poiseuille flow, the optimum dimensions of the structure compare very favorably to the length scales of the turbulent coherent structures found via direct numerical simulation (DNS) and experiment. ${ }^{26}$ Here, the optimum length scales correspond to the minimum Reynolds number at which the Newtonian ECS exist $\left(\operatorname{Re}_{\tau}\right.$ $=44.21$ for the case of channel flow). The optimum spanwise length, $L_{z}^{+}=105.51$, closely matches the streak spacing of one-hundred wall-units measured in experiments over a wide range of Reynolds numbers. ${ }^{9}$ (Throughout this work, the + superscript indicates that the length is scaled by the viscous length scale $\nu / u_{\tau}$, where the $u_{\tau}=\sqrt{\tau_{\text {wall }} / \rho}$ is the friction velocity and $\nu$ is the total, zero-shear-rate kinematic viscosity.) Minimal channel flow, i.e., flow in the smallest computational domain that reproduces the velocity field statistics of near-wall turbulence, gives a range for the streamwise length 
of 250-350, compared to $L_{x}^{+}=273.73$ for the ECS, and a spanwise length that is again approximately 100 wall units. ${ }^{33}$ The statistics of the near-wall region are faithfully captured in the minimal channel flow up to $y^{+} \approx 40$, while the wallnormal size of the optimum ECS is $\Delta y^{+}=44.21$. It should be pointed out that this minimum channel contains a single wavelength of a wavy streak and a pair of quasistreamwise vortices, which is the same structure seen in the ECS. Another invariant length scale in near-wall turbulence is the peak in the production of turbulent kinetic energy at $y^{+}$ $\approx 12 ;^{11}$ the channel flow ECS also captures this length scale. ${ }^{34}$ The optimum ECS quantitatively capture the length scales of near-wall turbulence.

Beyond capturing the observed length scales of the buffer region structures, recent research also indicates that the ECS are saddle-points in phase space around which the strange attractor ${ }^{29}$ of near-wall turbulence is built. Recent studies by Kawahara and $\mathrm{Kida}^{35}$ and Toh and Itano ${ }^{36}$ find periodic solutions in minimal channel flows that are bifurcations of the ECS. The bursting trajectories in fully turbulent flows seem to be built around these periodic solutions. A further indication of this comes from a study by Jiménez and Simens that applies a numerical filter to DNS of channel flow to isolate the near-wall region from the main-stream turbulence. $^{37}$ The simplest (nontrivial) flow structure, found when the numerical mask is at $y^{+} \approx 50$, is a traveling-wave solution that has qualitatively the same structure as the ECS. The length scales of this traveling wave $\left(L_{x}^{+} \approx 250, \Delta y^{+}\right.$ $\approx 50$, and $L_{z}^{+} \approx 150$ ) are similar to the optimum values for the channel flow ECS described above. As the mask moves further away from the wall, these traveling-wave solutions bifurcate into quasiperiodic solutions. These solutions then evolve into the bursts of full-scale turbulence with the flow being essentially turbulent when the numerical filter reaches $y^{+} \approx 70$. These results, along with the existence of the ECS indicate that staggered streamwise vortex traveling wave patterns are autonomous in wall-bounded shear flows and provide, at least in part, the foundation on which the near-wall turbulent fluctuations are built.

The self-sustaining process that underlies the ECS, making them autonomous, consists of three interacting, concurrent subprocesses: streak formation, streak instability, and vortex regeneration. ${ }^{38,39}$ The counter-rotating, streamwisealigned vortex pairs pull fluid with low streamwise velocity up from near the wall while, at the opposite side of a vortex, higher velocity fluid is pulled toward the wall. This leads to a spanwise stratification of the streamwise velocity (i.e., streaks in the streamwise velocity). The spanwise inflections in the streamwise velocity lead to a three-dimensional Kelvin-Helmholtz-like instability ${ }^{40}$ that concentrates wallnormal vorticity. This concentrated wall-normal vorticity, through a nonlinear interaction with the mean shear, is tilted and stretched in the streamwise direction, reenergizing the streamwise vortices. In studying the viscoelastic ECS, we aim to understand how the polymer affects one or all of the subprocesses that comprise this mechanism.

We have previously studied viscoelastic ECS with lowextensional-viscosity polymer solutions. ${ }^{41}$ That work provided some structural insight into the mechanism of drag reduction. The polymer molecules become highly elongated as they move through the streamwise streak. As they move out of the streak and into one of the vortices, the polymer molecules relax. This relaxation produces a polymer force that opposes the vortex motion. The polymer force thus weakens the vortex by slowing the fluid moving into it. The weakening of the vortices leads to collapse of the mechanism that sustains the ECS, ${ }^{39}$ and ultimately to a reduction in drag. This mechanism is also supported by elastic energy arguments proposed in a recent DNS study by Min et $a l .{ }^{42}$ In their study, they find that during drag reduction turbulent energy is transformed into elastic energy by the stretching of the polymer near the wall. The vortices of the buffer region then lift this elastic energy up where it is released as turbulent kinetic energy or dissipated in the buffer and log regions. In the present study, we study the viscoelastic ECS for high extensional viscosity solutions, mapping out how their existence region changes with ECS and tracking the changes in the flow structure both directly and through the Reynolds stress budgets. These results allow us to flesh out our previous work and show in some detail the mechanism by which the polymer leads to drag reduction and the associated changes in velocity statistics in this class of flows.

\section{MATHEMATICAL FORMULATION AND SIMULATION DETAILS}

The present study focuses on a variant of plane Couette flow. (The effects of viscoelasticity on the channel flow ECS will be addressed in subsequent studies.) Denoting the streamwise direction as $x$, the wall-normal direction as $y$, and the spanwise, or vorticity, direction as $z$, we consider a flow with boundary conditions

$$
\frac{\partial v_{x}}{\partial y}=1, \quad v_{y}=\frac{\partial v_{z}}{\partial y}=0 \text { at } y= \pm 1,
$$

where $v_{x}, v_{y}$, and $v_{z}$ are the streamwise, wall-normal, and spanwise components of the velocity, $\mathbf{v}$, respectively. The characteristic velocity $U$, which is the velocity of the unidirectional laminar solution at the upper boundary, and the half-height of the channel $l$ are used to scale the velocity and positions, respectively (though we will report results in wall units, with $y^{+}$measured from the bottom boundary $y=-1$ ). These "constant vorticity" boundary conditions provide an advantage over no-slip conditions in that they allow us to model only the buffer region in our domain by eliminating the viscous sublayer. The use of no-slip boundary conditions does not qualitatively change the behavior of the ECS. ${ }^{26}$ For this flow, a trivial base state exists, $\mathbf{v}(y)=y \mathbf{e}_{\mathbf{x}}$ for $-1 \leqslant y \leqslant 1$. Periodic boundary conditions are used in the streamwise and spanwise directions. For this study, the wavelength of the structures in the streamwise and spanwise directions are $L_{x}$ $=2 \pi / 0.40\left(L_{x}^{+} \approx 160\right.$ at $\left.\operatorname{Re}_{\tau}=20.5\right)$ and $L_{z}=2 \pi / 1.0\left(L_{z}^{+}\right.$ $\approx 64$ ), respectively.

In our formulation, time $t$ is scaled with $l / U$, and pressure $p$ with $\rho U^{2}$, where $\rho$ is the fluid density. The stress due to the polymer $\tau_{p}$ is nondimensionalized with the polymer elastic modulus $G=\eta_{p} / \lambda$, where $\eta_{p}$ is the polymer contribution to the zero-shear-rate viscosity and $\lambda$ is the time con- 
stant for the polymer-the polymer model is described below. For a traveling wave solution $\mathbf{v}\left(x-C_{v} t, y, z\right)$, where $C_{v}$ is the wave speed, the momentum balance and the equation of continuity are

$$
\begin{aligned}
-C_{v} \frac{\partial \mathbf{v}}{\partial x}+\mathbf{v} \cdot \boldsymbol{\nabla} \mathbf{v}= & -\boldsymbol{\nabla} p+\beta \frac{1}{\operatorname{Re}} \nabla^{2} \mathbf{v} \\
& +(1-\beta) \frac{1}{\operatorname{Re}} \frac{1}{\mathrm{We}}\left(\boldsymbol{\nabla} \cdot \boldsymbol{\tau}_{p}\right),
\end{aligned}
$$

$$
\boldsymbol{\nabla} \cdot \mathbf{v}=0,
$$

where $\eta_{s}$ is the solvent viscosity, $\mathrm{We}=\lambda U / l$ is the friction Weissenberg number, and $\beta=\eta_{s} /\left(\eta_{s}+\eta_{p}\right)$. The Reynolds number $\mathrm{Re}$ is based on the total zero-shear-rate viscosity, $\operatorname{Re}=\rho U l /\left(\eta_{s}+\eta_{p}\right)$ and we define the friction Reynolds number as the full height of the channel-i.e., the wall-normal extent of the streamwise vortices-expressed in wall units, so $\operatorname{Re}_{\tau}=2 \sqrt{\operatorname{Re}}$.

We seek "sinuous" solutions that satisfy the shift-reflect symmetry

$$
\begin{aligned}
& v_{x}(x, y, z)=v_{x}\left(x+L_{x} / 2, y,-z\right), \\
& v_{y}(x, y, z)=v_{y}\left(x+L_{x} / 2, y,-z\right), \\
& v_{z}(x, y, z)=-v_{z}\left(x+L_{x} / 2, y,-z\right),
\end{aligned}
$$

and the "Couette" symmetry

$$
\begin{aligned}
& v_{x}(x, y, z)=-v_{x}\left(L_{x} / 2-x,-y, z+L_{z} / 2\right), \\
& v_{y}(x, y, z)=-v_{y}\left(L_{x} / 2-x,-y, z+L_{z} / 2\right), \\
& v_{z}(x, y, z)=v_{z}\left(L_{x} / 2-x,-y, z+L_{z} / 2\right) .
\end{aligned}
$$

Solutions satisfying these latter three conditions travel with the mean velocity of the trivial solution so they have wave speed $C_{v}=0$ independent of Re or We.

We calculate the polymer stress using an idealized polymer model, a solution of elastic dumbbells. Each molecule consists of two beads, where the mass and drag of the molecule are concentrated, connected by a finitely extensible nonlinear elastic (FENE) spring. An approximation of the FENE spring, introduced by Peterlin, provides a closed constitutive equation for the conformation of the dumbbell. This is the FENE-P model, ${ }^{43}$ which, written for a solution $\boldsymbol{\alpha}(x$ $\left.-C_{v} t, y, z, t\right)$ becomes

$$
\begin{aligned}
& \frac{\boldsymbol{\alpha}}{1-\frac{\operatorname{tr} \boldsymbol{\alpha}}{b}}+\operatorname{We}\left(\frac{\partial \boldsymbol{\alpha}}{\partial t}-C_{v} \frac{\partial \boldsymbol{\alpha}}{\partial x}+(\mathbf{v} \cdot \boldsymbol{\nabla} \boldsymbol{\alpha})-\{\boldsymbol{\alpha} \cdot \boldsymbol{\nabla} \mathbf{v}\}\right. \\
& \left.-\{\boldsymbol{\alpha} \cdot \boldsymbol{\nabla} \mathbf{v}\}^{T}\right)=\left(\frac{b}{b+2}\right) \boldsymbol{\delta}, \\
& \boldsymbol{\tau}_{p}=\frac{b+5}{b}\left[\frac{\boldsymbol{\alpha}}{1-\frac{\operatorname{tr} \boldsymbol{\alpha}}{b}}-\left(1-\frac{2}{b+2}\right) \boldsymbol{\delta}\right],
\end{aligned}
$$

where $\boldsymbol{\alpha}$ is the nondimensional conformation tensor and $b$ is proportional to the contour length of the polymer- $\operatorname{tr} \boldsymbol{\alpha}$ cannot exceed $b$. Many authors use a slightly different form of the FENE-P model, in which the factors $b /(b+2),(b+5) / b$, and $2 /(b+2)$ in the above equations are replaced by 1,1 , and 0 , respectively. This difference is negligible in the present situation, where $b>10^{3}$.

It is well-recognized that extensional rheology plays a key role in turbulent drag reduction. A simple measure of the importance of extensional polymer stress is the magnitude of the parameter

$$
\mathrm{Ex}=\frac{2}{3} \frac{b \eta_{p}}{\eta_{s}}=\frac{2}{3} \frac{b(1-\beta)}{\beta} .
$$

This parameter represents the ratio between viscous and polymer stresses in extensional flow, and its definition follows from the asymptotic expansion for high elongation rates of the extensional viscosity for the FENE dumbbell model. ${ }^{43}$ In uniaxial extension with extension rate $\dot{\varepsilon}$,

$$
\begin{aligned}
\mathrm{Ex}=1 & \Leftrightarrow \tau_{p}=\tau_{v} \text { as } \dot{\varepsilon} \rightarrow \infty, \\
& \Leftrightarrow \eta^{+}=3 \eta_{s} \text { as } \dot{\varepsilon} \rightarrow \infty,
\end{aligned}
$$

where $\boldsymbol{\tau}_{v}$ is the solvent contribution to the stress and $\eta^{+}$is the extensional viscosity of the solution. Since turbulent flows, as well as the ECS that we study here, are extensional on average, one would expect to see a significant effect of the polymer on the flow once $E x \gtrsim 1$. For real drag-reducing solutions, Ex values can be orders of magnitude greater than 1. For example, our results in this study are for $\mathrm{Ex}=100$ and $\beta=0.97$, which corresponds to $b=4850$. This value of $b$ corresponds to a solution of $250000 \mathrm{MW}$ polyethylene oxide in water. Since under the conditions studied here, polymer extensions in the viscoelastic ECS are generally much less than the maximum possible extension (see Fig. 10 and related discussion), we do not expect the results to qualitatively change at higher Ex values.

Given the relative simplicity of the FENE-P model compared to real polymers, a natural question is its fidelity to real polymer dynamics in complex flows. To address this point, we have previously compared FENE-P to more realistic bead-spring-chain models for calculating the stresses in the flow field of a Couette flow ECS. ${ }^{44}$ We performed Brownian dynamics simulations of 20-spring chains, with hydrodynamic and excluded volume interactions among the polymer segments explicitly included, as they moved along Lagrangian trajectories in the ECS. Comparing the FENE-P results to the "exact" results from the chain models, we found that the FENE-P model qualitatively captured the spatial variations in polymer stress. The largest quantitative differences in the stresses were seen when the polymer chains were stretched to near their full extension. For the $b$ value and Weissenberg number range used in the present study the polymer stretch in the viscoelastic ECS is generally less than $30 \%$ of the full extension (again, see Fig. 10 and related discussion). For these low relative levels of polymer extension, only small quantitative differences between FENE-P and the beadspring-chain models were seen. For these reasons, we believe that the FENE-P model will adequately capture the effects of polymers on the ECS in the parameter range studied here.

The conservation and constitutive equations are solved through a Picard iteration. The velocity field of a Newtonian 
ECS, as computed in Ref. 24 (or, for higher Ex values, a viscoelastic solution found for lower We or Ex values), is first used to calculate the polymer stress tensor $\tau_{p}$ by inserting the velocity field in the evolution equation for $\boldsymbol{\alpha}$ and integrating for a short length of time, usually one time unit $(l / U)$. For this $\tau_{p}$, a steady state of the momentum and continuity equations is found by Newton iteration. The resulting velocity field $\mathbf{v}$ is used to compute the new $\boldsymbol{\tau}_{p}$, and the process is repeated until the velocity and polymer stress fields converge to a steady state (in a reference frame traveling with the wave speed). This Picard iteration, while not optimal, obviates a large-scale Newton iteration $\left(\sim 10^{6}\right.$ unknowns in a nonsymmetric, indefinite problem).

The momentum and continuity equations are discretized using a Fourier-Galerkin formulation with typically a 7 $\times 19 \times 7$ grid. The conformation tensor $\boldsymbol{\alpha}$ is discretized with a third-order, compact upwind difference scheme ${ }^{45}$ (see also Refs. 46 and 47 for applications of compact differences to polymer drag reduction). In this, as in most previous computational studies of polymers in turbulent flows, we have found it necessary to add an artificial stress diffusivity, $(1 / \mathrm{ScRe}) \nabla^{2} \boldsymbol{\alpha}$, to Eq. (4) to achieve numerical stability. The Schmidt number $\mathrm{Sc}=\nu / D$, which is the ratio of the momentum diffusivity to stress diffusivity, is set to a value of 1 . This value of Sc, though artificially small, is greater or of the same order of magnitude as that used in many DNS studies. $^{12,17,48,49}$ In the range of Sc where solutions can be obtained, the bifurcation diagrams shown below are very insensitive to its value. The stress diffusion term is integrated implicitly by the Crank-Nicholson method with the other terms of the equation integrated using the Adams-Bashforth method. This equation is solved on a finer mesh than the momentum, continuity pair, typically $32 \times 32 \times 32$. Higher resolutions $(8 \times 21 \times 8$ for the momentum, continuity pair and $48 \times 48 \times 48$ for the polymer stress) show less than $0.1 \%$ change in the Fourier amplitudes of the velocity compared to the lower resolutions.

\section{RESULTS}

\section{A. Existence of the ECS}

Throughout this study, we analyze the Newtonian and viscoelastic exact coherent states at fixed streamwise and spanwise lengths: $L_{x}=2 \pi / 0.4$ and $L_{z}=2 \pi\left(L_{x}^{+} \approx 160\right.$ and $L_{z}^{+}$ $\approx 64$ at $\left.\operatorname{Re}_{\tau}=20.5\right)$. The bifurcation diagram, which shows the solutions of Eqs. (2) and (3) as Re increases, is given in Fig. 2. These solutions are plotted using the maximum in the rms wall-normal velocity fluctuations for the solution, $\overline{v_{y}^{\prime 2}}{ }^{1 / 2}$. (Hereafter, an overbar indicates that the variable is averaged over the streamwise and spanwise directions.) The trivial base state in this geometry $[\mathbf{v}(y)=(y, 0,0)]$ exists at all $\operatorname{Re}$ and would appear as a horizontal line at $\max \overline{v_{y}^{\prime 2}} 1 / 2=0$ if it were plotted in Fig. 2. At $\operatorname{Re} \approx 105\left(\operatorname{Re}_{\tau} \approx 20.5\right)$ for the Newtonian flow (solid curve), two new solutions that have nonzero wall-normal velocity appear via a saddle-node bifurcation. These are the ECS. The solutions with higher maximum wall-normal fluctuations at a given Re, i.e., solutions on the upper branch of the bifurcation diagram, we call "high drag" solutions due to their lower mean velocity at $y=1$ compared

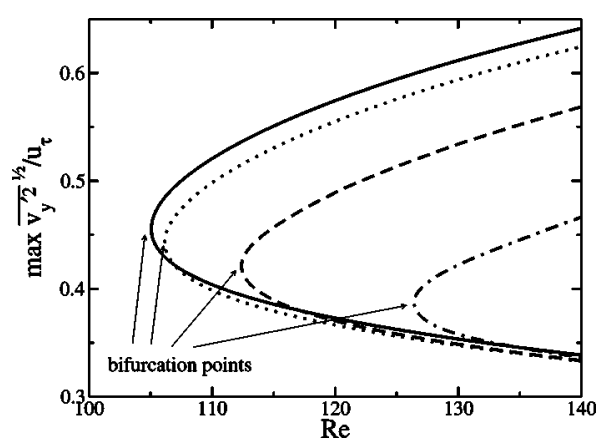

FIG. 2. Bifurcation diagram for Newtonian and viscoelastic ECS with $L_{x}$ $=2 \pi / 0.4, L_{z}=2 \pi$ and for the viscoelastic solutions $\operatorname{Ex}=100(b=4850)$ and $\beta=0.97$. - , Newtonian, ..., $\mathrm{We}=7 ;---, \mathrm{We}=9 ;---, \mathrm{We}=11$.

to the lower branch, or "low drag," solutions. The "high drag" ECS are more important to the dynamics of the turbulent coherent structures, as explained in Sec. I, and are the main focus of this study. All results should be assumed to be for the "high drag" states unless otherwise indicated.

For the viscoelastic flow at $\mathrm{We}=7$, the dotted curve in Fig. 2, the ECS again appear via a saddle node bifurcation. However, the bifurcation occurs at a Reynolds number, $\operatorname{Re}_{\min } \approx 107\left(\operatorname{Re}_{\tau} \approx 20.7\right)$, that is higher than the Re for the appearance of the Newtonian ECS. As the Weissenberg number increases to $\mathrm{We}=9$ (dashed curve) and $\mathrm{We}=11$ (dashdotted curve), the Re where the bifurcations occur likewise increase to $\operatorname{Re} \approx 112 \quad\left(\operatorname{Re}_{\tau} \approx 21.2\right)$ and $\operatorname{Re} \approx 127 \quad\left(\operatorname{Re}_{\tau}\right.$ $\approx 22.4$ ), respectively. Accompanying this change in the Re at which the bifurcation occurs are changes to the flow field as indicated by the decrease in the maximum of the rms wallnormal velocity fluctuations at a given Re as We increases. This change in the wall-normal velocity is more dramatic for the "high drag" state than for the "low drag" state. Figure 3 shows the streamwise velocity for both the "high drag" and "low drag" states at $y^{+}=11$ (i.e., on a plane parallel to the walls). Since the "low drag" ECS have weaker streamwise vortices and are essentially less "three-dimensional," i.e., the streamwise streaks have a weaker $x$-dependence, they are less effective than the "high drag" states at stretching the

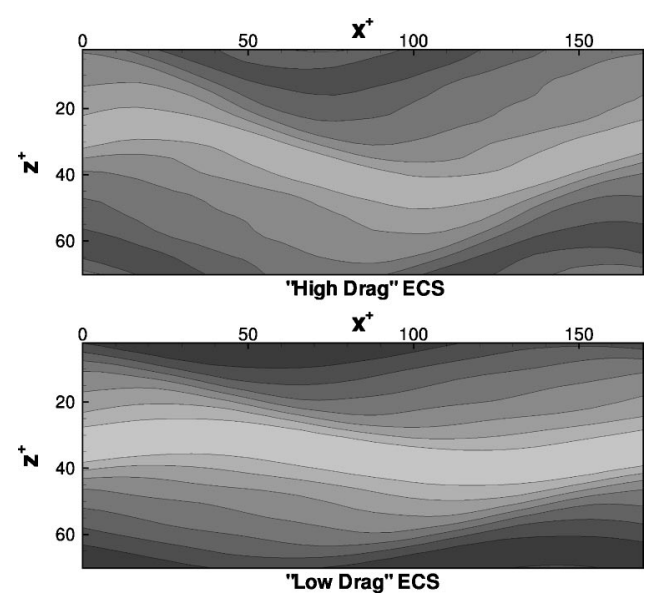

FIG. 3. Streamwise velocity contours for the "high drag" and "low drag" exact coherent states for $\operatorname{Re}=124$ at $y^{+}=11$ (the center of the channel). Range: $v_{x}^{+}=7.8$ (light gray) and $v_{x}^{+}=-7.8$ (dark gray). 


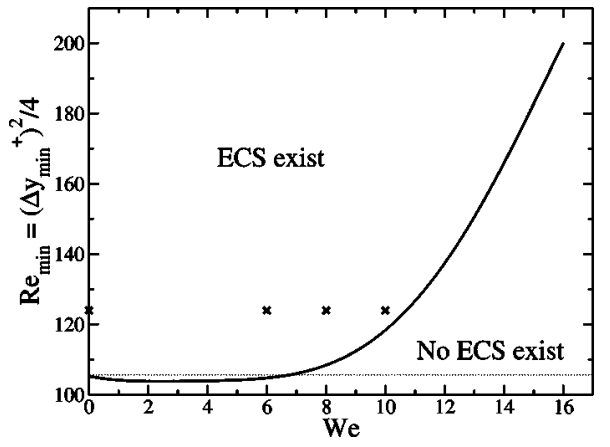

FIG. 4. Change in the minimum $\operatorname{Re}\left(=\left(\Delta y^{+}\right)^{2} / 4\right)$ as We increases. $L_{x}$ $=2 \pi / 0.4, L_{z}=2 \pi, \mathrm{Ex}=100$, and $\beta=0.97$. The dotted line shows the minimum Re for the Newtonian ECS at the same streamwise and spanwise wavelengths. The solutions studied in Figs. 11 and 12 are marked by $\times$ 's.

polymer at a given We, leading to a smaller change in max $v_{y}^{\prime 2} 1 / 2$ in the "low drag" states. Further results for the rms wall-normal velocity fluctuations and other statistics of the flow will be presented and interpreted subsequently.

To gain a better understanding of how the existence of the ECS is affected by the addition of polymer, we look at how the minimum value of Reynolds number at which the ECS can exist, $\mathrm{Re}_{\min }$ (the bifurcation points in Fig. 2), changes with Weissenberg number. This curve of $\mathrm{Re}_{\min }$, given in Fig. 4, separates the region where the ECS can exist (above the curve) from the region where no ECS exist. At low We, there is only a small difference between the $\mathrm{Re}_{\text {min }}$ values for the Newtonian and viscoelastic ECS. At $\mathrm{We} \approx 7$ the minimum $\mathrm{Re}$ for the viscoelastic ECS increases above that for the Newtonian. As Weissenberg number increases further, there is a dramatic increase in $\mathrm{Re}_{\min }$ with the bifurcation point for $\mathrm{We}=16$ appearing at a Reynolds number almost twice that of the Newtonian ECS. The importance of this increase in $\mathrm{Re}_{\min }$ is seen more easily when one notes that the Reynolds number is simply related to the wall-normal length scale of the structure measured in wall units, $\Delta y^{+}$, by the relation $\operatorname{Re}=\left(\Delta y^{+}\right)^{2} / 4$. In experiments, the thickness of the buffer region (the wall-normal extent of this region in wall units) is known to increase as drag reduction increases. The results for the viscoelastic ECS closely mirror this increase in $\Delta y^{+}$. For this reason, we will refer to the We above which the critical value of $\Delta y^{+}$for the viscoelastic ECS is greater than for the Newtonian ECS as the onset Weissenberg number $\mathrm{We}_{\text {onset }}$. As seen in Fig. 4, $\mathrm{We}_{\text {onset }} \approx 7$, which agrees well with onset values from two recent DNS studies of polymer drag reduction, ${ }^{19,42}$ which find onset at $\mathrm{We} \approx 6$. This close agreement in $\mathrm{We}_{\text {onset }}$ is perhaps somewhat fortuitous since the DNS studies are for fully turbulent channel flows, not the Couette ECS flows studied here.

The dramatic increase in $\mathrm{Re}_{\min }$ once $\mathrm{We} \gtrsim 7$ may also be related to the observation that transition to turbulence in a polymer solution is delayed to higher Re than in the Newtonian case. ${ }^{8,14}$ Evidence suggests that the existence of ECS is a prerequisite for transition (cf. the Introduction). If this is the case, then an increase in the Reynolds number at which the ECS exist implies a corresponding increase in the transition Reynolds number, as is found experimentally.

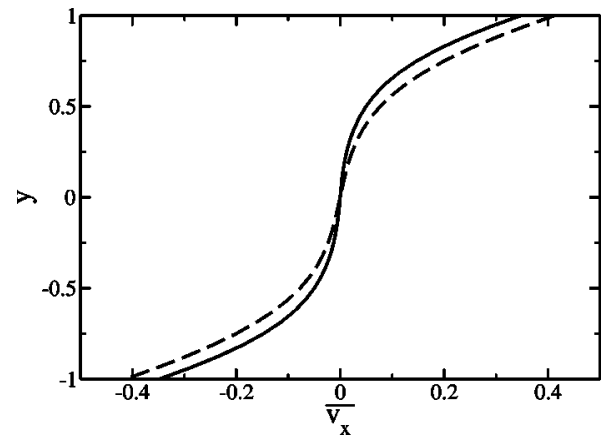

FIG. 5. Mean streamwise velocity: - , Newtonian- $\operatorname{Re}=200$; - - viscoelastic $-\mathrm{Re}=200, \mathrm{We}=16.0, \mathrm{Ex}=100$, and $\beta=0.97$.

Given what we have learned about the existence of the ECS, we now look in detail at what happens to the ECS at constant Reynolds number as the Weissenberg number increases. The mean velocity profiles for the Newtonian and viscoelastic flows at $\mathrm{Re}=200(\mathrm{Re}=28.3)$ are given in Fig. 5 . These mean profiles show the typical "S-shaped" curve seen in fully turbulent plane Couette flows. The maximum mean velocity in the ECS is reduced compared to the base state velocity due to enhanced redistribution of the streamwise momentum by the streamwise vortices. For the viscoelastic ECS, the mean velocity increases significantly compared to the Newtonian, illustrating that drag is reduced by the addition of polymer. For the case in Fig. $5(\mathrm{Re}=200, \mathrm{We}=16$, $\mathrm{Ex}=100$, and $\beta=0.97$ ), drag is reduced by roughly $8 \%$. Note that at a slightly higher We, the ECS cannot exist at all at this Reynolds number $-\mathrm{Re}=200$ is very near the turning point at $\mathrm{We}=16$. The changes to the streamwise velocity fluctuations, scaled by the friction velocity $u_{\tau}$ are given in Fig. 6 . Keep in mind that the boundary conditions used in this simulation eliminate the viscous sublayer, so $y^{+}=0$ is (roughly) the start of the buffer region. Near the lower edge of the buffer region, the streamwise velocity fluctuations are slightly smaller for the viscoelastic flow, but over most of the domain these velocity fluctuations increase. This result mirrors observations in fully turbulent drag reduced flows and is analyzed to a greater extent in Sec. III B.

Instead of examining how the velocity fluctuations change over the whole flow domain as in Figs. 5 and 6, we

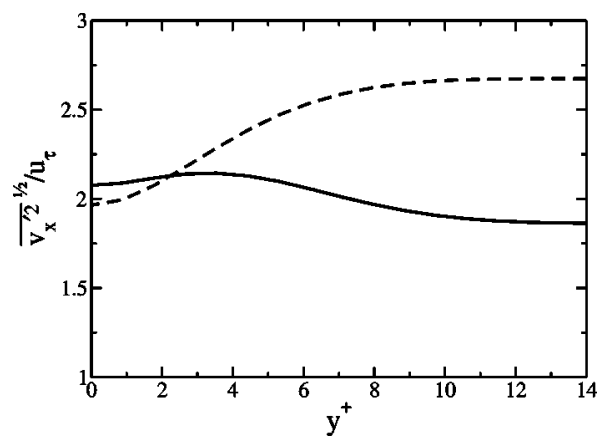

FIG. 6. Fluctuations in the streamwise velocity: - , Newtonian- $\operatorname{Re}=200$; --- , viscoelastic $-\mathrm{Re}=200, \mathrm{We}=16.0, \mathrm{Ex}=100$, and $\beta=0.97$. Only half of the channel is shown; the other half is simply a reflection across the centerline due to the symmetry of Couette flow. 


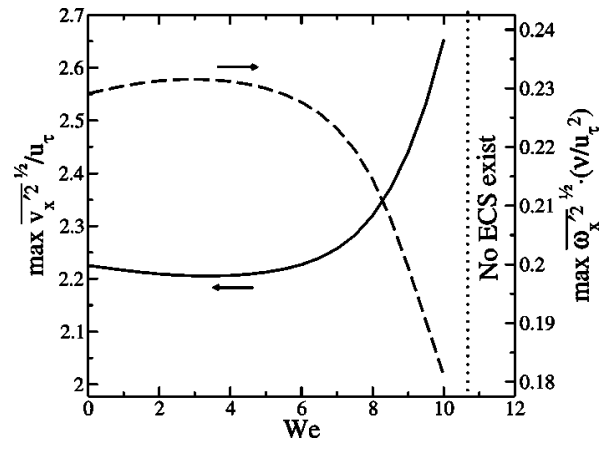

FIG. 7. Maxima in the streamwise- and spanwise-averaged fluctuations in streamwise velocity (-) and streamwise vorticity $(---)$ vs We for $\operatorname{Re}$ $=124, \mathrm{Ex}=100$, and $\beta=0.97$.

now look at how the maximum (or minimum) over the domain of a flow variable changes as We increases, at constant Re. Figure 7 gives such a plot for the streamwise velocity and vorticity fluctuations at $\mathrm{Re}=124$. At low We, there is very little change to the maximum values of these variables compared to the Newtonian. However, as the Weissenberg number rises above $\mathrm{We}_{\text {onset }}$, the streamwise velocity fluctuations increase rapidly while the streamwise vorticity decreases similarly. The increase (decrease) of streamwise velocity (vorticity) gets steeper as the Weissenberg number approaches where the ECS cease to exist for $\mathrm{Re}=124$ (see Fig. 4). Similar results are seen for the wall-normal and spanwise velocity fluctuation (Fig. 8). For We smaller than the onset value, $v_{y}^{\prime 2} 1 / 2$ and $\max v_{z}^{\prime 2} 1 / 2$ deviate only slightly from the Newtonian values. Above this onset value, both wallnormal and spanwise fluctuations decrease drastically. Since the magnitudes of both $v_{y}^{\prime}$ and $v_{z}^{\prime}$ are greatest at the edges of the streamwise vortices, we infer that their decrease is due to the weakening of the vortices, which is consistent with the rapid decrease of the streamwise vorticity. The changes in both $v_{x}^{\prime}$ and $v_{y}^{\prime}$ affect the Reynolds shear stress, $-\rho \overline{v_{x}^{\prime} v_{y}^{\prime}}$. The Reynolds shear stress is the wall-normal flux of streamwise momentum and, thus, is the drag due to the velocity fluctuations. Figure 9 shows that the reduction in Reynolds shear stress is slightly more pronounced at lower We than is seen for the velocity fluctuations. Above $\mathrm{We}_{\text {onset }},-\overline{v_{x}^{\prime} v_{y}^{\prime}}$ decreases dramatically as we saw with the velocity fluctuations. Since the Reynolds shear stress is the key component of the drag,

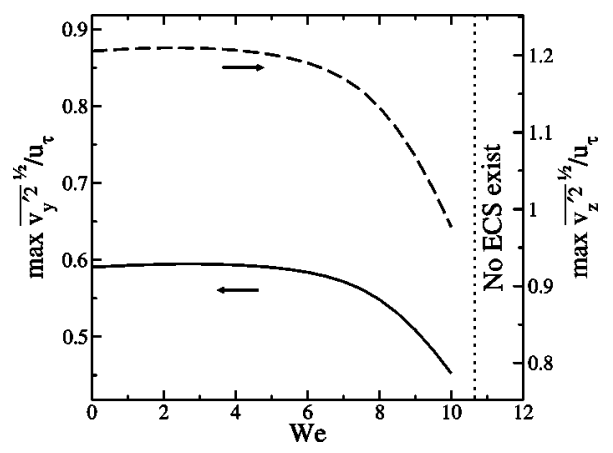

FIG. 8. Maxima in the streamwise- and spanwise-averaged fluctuations in wall-normal velocity (-) and spanwise velocity (---) vs We for $\mathrm{Re}$ $=124, \mathrm{Ex}=100$, and $\beta=0.97$.

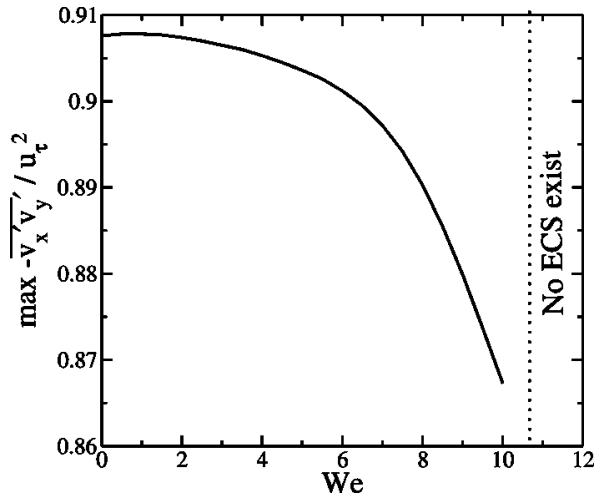

FIG. 9. Maximum in the Reynolds shear stress vs We for $\mathrm{Re}=124$, Ex $=100$, and $\beta=0.97$.

this result provides a further indication of drag reduction in the viscoelastic ECS. The analysis of the Reynolds stress budgets in Sec. III B will shed more light on this reduction of Reynolds shear stress.

If the polymers are suppressing the vortices, as indicated by decreases in $v_{y}^{\prime}, v_{z}^{\prime}$, and $\omega_{x}^{\prime}$, then we can expect to see changes in the pressure fluctuations $\left(p^{\prime}\right)$ because they reach their greatest magnitude (i.e., they are most negative) in the vortex cores. Figure 10 shows the minimum of the pressure fluctuations versus We. From this plot, we again see that above $\mathrm{We}_{\text {onset }}$, there is a significant change in the flow that causes the minimum of the pressure fluctuations to increase. Since $p^{\prime}$ is most negative in the cores, this increase in $p^{\prime}$ must be due to the weakening of the vortices. This result can be more easily seen in a plot of isosurfaces of the pressure fluctuations (Fig. 11) with $p^{\prime}$ equal to $60 \%$ of the minimum $p^{\prime}$ for the Newtonian ECS at $\mathrm{Re}=124$. By comparing this figure to Fig. 1, it is obvious that the isosurfaces of negative $p^{\prime}$ correspond to the cores of the streamwise vortices. As the Weissenberg number increases, the isosurfaces become smaller, indicating that the strength of the vortices is diminishing. As will be shown in Sec. III B, this decrease in the magnitude of the pressure fluctuations has a crucial impact on the budgets for the Reynolds stresses. Isosurfaces of constant streamwise vorticity, seen in Fig. 12, corroborate the results from the pressure fluctuations. As We increases, the $\omega_{x}$ surfaces grow smaller, consistent with vortex suppression.

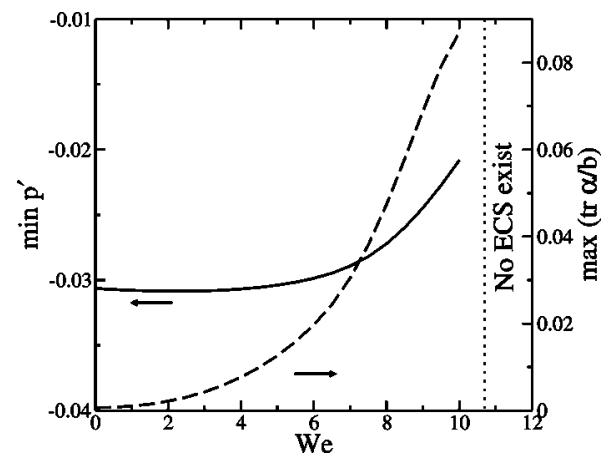

FIG. 10. Minimum in the pressure fluctuations (-) and maximum in the trace of the conformation tensor, $\boldsymbol{\alpha},(---)$ vs We for $\operatorname{Re}=124, \mathrm{Ex}=100$, and $\beta=0.97$. 

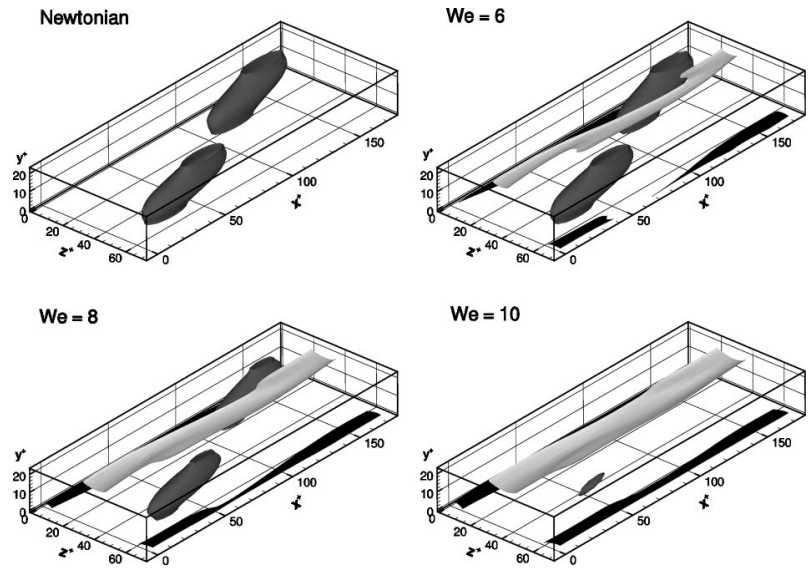

FIG. 11. Isosurfaces of pressure fluctuations, $p^{\prime}=0.60 p_{N \text { min }}^{\prime}$ (dark gray), and streamwise polymer force $\left(\boldsymbol{\nabla} \cdot \boldsymbol{\tau}_{p}\right)_{x}=-0.0027$ (white) and 0.0027 (black). These values of the polymer force are $60 \%$ of the minimum and maximum values of the force at $\mathrm{We}=6 . \operatorname{Re}=124\left(\operatorname{Re}_{\tau}=22.27\right), \mathrm{Ex}=100$, and $\beta=0.97$. Top left: Newtonian; top right: $\mathrm{We}=6$; bottom left: $\mathrm{We}=8$; bottom right: $\mathrm{We}=10$.

Understanding that the salient effect of viscoelasticity on the ECS is the suppression of the streamwise vortices, we can investigate how this is brought about by studying the spatial distributions of the polymer stress $\tau_{p}$ and, more importantly, the force exerted by the polymer on the flow $\mathbf{f}$ $=[(1-\beta) / \mathrm{ReWe}] \boldsymbol{\nabla} \cdot \boldsymbol{\tau}_{p}$. Figure 13 shows the streamwise- and spanwise-averaged trace of the polymer stress tensor. The polymer stress is higher near the edge of the domain with the maximum occurring at $y^{+} \approx 2$. (By symmetry identical behavior is occurring near the upper boundary.) This spatial position for the maximum in polymer stress coincides with the streak in the streamwise velocity, indicating that the polymer molecules are highly stretched as they move through the streamwise streak. Even though the polymers are highly stretched compared to their equilibrium length, their end-toend distance generally remains well below their contour length. As shown in Fig. 10, the maximum in the stretch polymer $(\operatorname{tr} \boldsymbol{\alpha})$ is less than $10 \%$ of its greatest possible extension $(b)$. Eventually, these highly stretched molecules flow
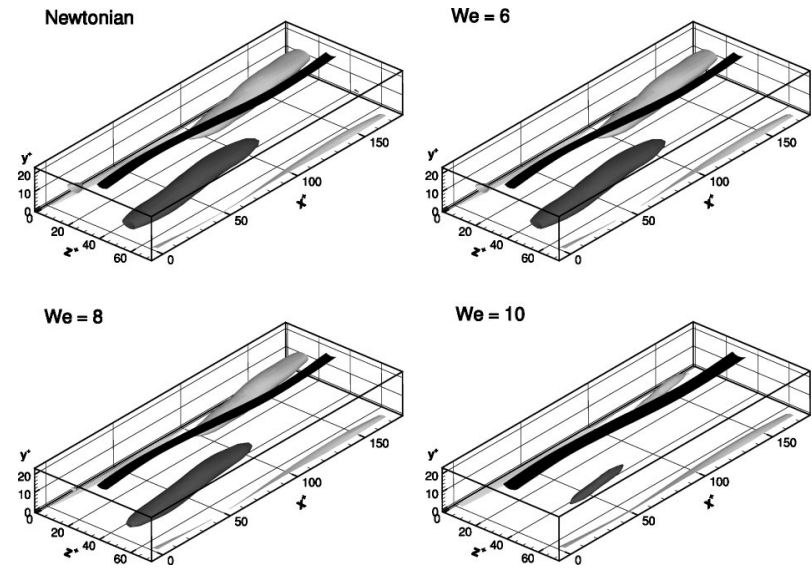

FIG. 12. Isosurfaces of streamwise vorticity, $\omega_{x}^{+}=0.41$ (light gray) and $\omega_{x}^{+}$ $=-0.41$ (dark gray), and streamwise velocity, $v_{x}^{+}=-8.5$ (white), and $v_{x}^{+}$ $=8.5$ (black). $\operatorname{Re}=124\left(\operatorname{Re}_{\tau}=22.27\right), \mathrm{Ex}=100$, and $\beta=0.97$. Top left: Newtonian; top right: $\mathrm{We}=6$; bottom left: $\mathrm{We}=8$; bottom right: $\mathrm{We}=10$.

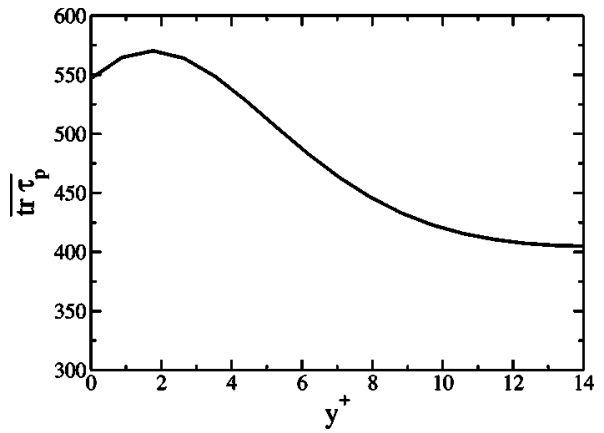

FIG. 13. Streamwise- and spanwise-averaged polymer stress in the viscoelastic $\mathrm{ECS}$. $\mathrm{Re}=200, \mathrm{We}=16.0, \mathrm{Ex}=100$, and $\beta=0.97$.

out of the streaks and toward the channel centerline in the upwellings at the edges of the streamwise vortices. Since the polymer stress seen in Fig. 13 decreases toward the centerline, these molecules must be relaxing as they move into and around the vortices. (This process of polymer molecules stretching in the streaks and relaxing at the edges of the vortices is also confirmed by Brownian dynamics simulations of model polymers along a Lagrangian trajectory in the ECS. $^{44}$ ) The result of this process of stretching and relaxation of the polymer is a region of high polymer stress in the streamwise streak and a region of lower polymer stress where fluid elements have left the streaks and begun to move around one of the vortices. Taking the divergence of the polymer stress gives a force due to the polymer that is directly opposed to the movement of fluid into the vortex. Since the fluid elements moving into the vortex are being slowed by the polymer force, the vortex is weakened. This vortex suppression leads to the results described in the preceding paragraphs.

As a further indication of the origin of the vortex suppression, Fig. 14 shows a comparison of the wall-normal and spanwise velocities to the corresponding components of the polymer force. The upwelling and downwelling associated with the counter-clockwise rotating vortex $\left(\omega_{x}<0\right)$ are clearly seen in the right half of the $v_{y}$ plot. The regions where the wall-normal and spanwise velocities are highest (white contours) are matched by the negative regions of the polymer force (black contours) and, likewise, negative regions of $v_{y}$ and $v_{z}$ correspond to positive $f_{y}$ and $f_{z}$. Figure 11 shows isosurfaces of the $x$-component of the polymer force at Re $=124$. Comparing these isosurfaces to those for the stream-
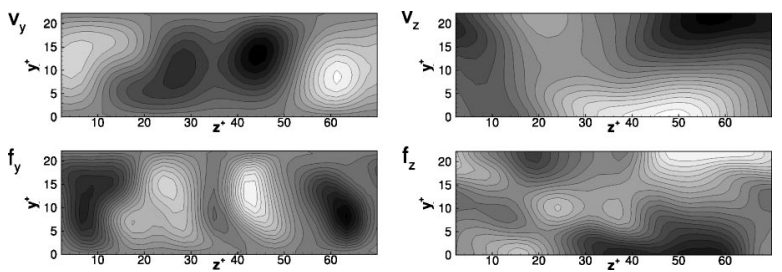

FIG. 14. Contours of velocity and polymer force at $x^{+}=38$ for the viscoelastic ECS with $\mathrm{Re}=124, \mathrm{We}=10, \mathrm{Ex}=100$, and $\beta=0.97$. Top left: $v_{y}^{+}$, range -0.96 (black) -0.96 (white) top right: $v_{z}^{+}$, range -2.14 (black) -2.14 (white) bottom left: $f_{y}$, range -0.00055 (black) -0.00055 (white) bottom right: $f_{z}$, range -0.0010 (black) -0.0010 (white). 
wise velocity given in Fig. 12, we see that they do occur at the same spatial positions and that they are anticorrelated (i.e., $f_{x}$ is negative where $v_{x}$ is positive and vice versa). Nevertheless, although the $x$-component of the polymer force is larger in magnitude than the other two components, $f_{y}$ and $f_{z}$ are more important in the suppression of the vortices and in drag reduction. In simulations where $f_{x}$ is artificially set to zero, we still see levels of drag reduction comparable to those seen when all three components are included. This is because $f_{y}$ and $f_{z}$ directly counteract the fluid motion in the streamwise vortices. This anticorrelation of polymer has also been found in the buffer layer via DNS of drag-reducing solutions. $^{50}$

The mechanism by which the polymer suppresses the streamwise vortices can be summarized as follows: Polymer molecules become stretched in the streamwise streak, then relax as they move from the streak into one of the streamwise vortices. The relaxation of the polymer molecules produces a force that directly opposes the motion of the fluid in the vortex and thus suppresses it. Recall from the Introduction that the mechanism that underlies the ECS consists of three subprocesses: streak formation by the streamwise vortices, streak instability, and vortex regeneration. The lowspeed streak in the flow is formed when the counter-rotating streamwise vortices pull slow moving fluid up from the lower boundary. Since the vortices are weakened by the polymer, the extent to which slow moving fluid is pulled up decreases. If the streak instability is weakened then the streamwise vortices are further weakened and the selfsustaining process tends to collapse.

\section{B. Effects on Reynolds stress budgets}

To gain more insight into how vortex suppression affects the velocity fields, we examine the budgets of the Reynolds stress balance. In their study of a fully turbulent channel flow with polymer injection at the wall, Walker and Tiederman ${ }^{7}$ found reduced levels of $v_{y}^{\prime}$ and $\overline{v_{x}^{\prime} v_{y}^{\prime}}$ and increased levels of $v_{x}^{\prime}$ due to the polymer. Since energy transfer among these quantities is represented by the pressure-strain correlations in the Reynolds stress budgets (as described below), they concluded that the polymer alters the processes represented by these correlations. A DNS study of polymer drag reduction corroborates this conclusion. ${ }^{18}$ In light of these results, we study the Reynolds stress budgets in the viscoelastic ECS to understand how the mechanism of drag reduction educed in the previous section inhibits the redistribution processes of the pressure-strain correlations. In the ECS, the polymer becomes highly stretched in the streamwise streaks of the turbulent coherent structures and relaxes as it moves into and around one of the streamwise vortices flanking the streak. This relaxation works to "unwind" the vortex and reduce its strength. Since the mechanism that sustains the coherent structures ${ }^{39}$ depends on the vortices to regenerate the streamwise streak, the suppression of the streamwise vortices leads to a collapse of this mechanism and ultimately to drag reduction. This section looks at the Reynolds stress budgets, in particular, to understand how the polymer affects the pressure-strain correlations, giving rise to the decreases in the Reynolds shear stress, $-\rho \overline{v_{x}^{\prime} v_{y}^{\prime}}$, and wall-normal velocity fluctuations and increases in streamwise velocity fluctuations.

The Reynolds stresses are actually $-\rho \overline{v_{i}^{\prime} v_{j}^{\prime}}$ but it is convenient and conventional to refer to $\overline{v_{i}^{\prime} v_{j}^{\prime}}$ as the Reynolds stresses, which we will do henceforth. In this section, we scale the Reynolds stresses with $u_{\tau}^{2}$ and time with $\nu / u_{\tau}^{2}$, where $u_{\tau}$ is the friction velocity and $\nu$ is the zero-shear-rate kinematic viscosity of the solution. The equation for the Reynolds stresses for a polymer solution can be written as

$$
\frac{\partial \overline{v_{i}^{\prime} v_{j}^{\prime}}}{\partial t}+\bar{V}_{k} \frac{\partial}{\partial x_{k}} \overline{v_{i}^{\prime} v_{j}^{\prime}}+T_{i j}^{t}=P_{i j}+D_{i j}+R_{i j}+T_{i j}^{p}+\varepsilon_{i j}+E_{i j},
$$

where the overbar represents an average over the streamwise and spanwise directions (cf. Ref. 11, Chap. 7). Here the velocity, pressure, and force due to the polymer $\left(\mathbf{f}=[(1-\beta) / \mathrm{ReWe}] \boldsymbol{\nabla} \cdot \boldsymbol{\tau}_{p}\right)$ are written as sums of mean and fluctuating parts $\left(\mathbf{v}=\overline{\mathbf{V}}+\mathbf{v}^{\prime}, p=\bar{P}+p^{\prime}\right.$, and $\left.\mathbf{f}=\overline{\mathbf{F}}+\mathbf{f}^{\prime}\right)$. The first two terms of Eq. (9) are zero for the ECS since they only include $x$-derivatives of the averaged quantities $\left[(\partial / \partial t) \frac{\partial v_{i}^{\prime} v_{j}^{\prime}}{v_{i}^{\prime} v_{j}^{\prime}}\right.$ $\rightarrow-C_{v}(\partial / \partial x) \overline{v_{i}^{\prime} v_{j}^{\prime}}=0$ and $\left.\bar{V}_{x}(\partial / \partial x) \overline{v_{i}^{\prime} v_{j}^{\prime}}=0\right]$. The terms

$$
T_{i j}^{t}=\frac{\partial}{\partial x_{k}} \overline{v_{i}^{\prime} v_{j}^{\prime} v_{k}^{\prime}}
$$

and

$$
T_{i j}^{p}=-\frac{\partial}{\partial x_{k}}\left(\overline{v_{i}^{\prime} p^{\prime}} \delta_{j k}+\overline{v_{j}^{\prime} p^{\prime}} \delta_{i k}\right)
$$

are the transport of Reynolds stress by the fluctuating velocities and the fluctuating pressure, respectively. Note that $T_{11}^{p}$ $=0$ since there are no gradients of the averaged quantities in the streamwise $x$ direction. The production term,

$$
P_{i j}=-\overline{v_{i}^{\prime} v_{k}^{\prime}} \frac{\partial \bar{V}_{j}}{\partial x_{k}}-\overline{v_{j}^{\prime} v_{k}^{\prime}} \frac{\partial \bar{V}_{i}}{\partial x_{k}},
$$

generates Reynolds stresses through interaction with the mean velocity gradient. For shear flows, which have $\overline{\mathbf{V}}$ $=\left[V_{x}(y), 0,0\right]$, the terms $P_{22}$ and $P_{33}$ are zero. Thus, there is no production of wall-normal or spanwise velocity fluctuations due to the mean shear. The pressure-rate-of-strain term

$$
R_{i j}=\overline{p^{\prime}\left(\frac{\partial v_{i}^{\prime}}{\partial x_{j}}+\frac{\partial v_{j}^{\prime}}{\partial x_{i}}\right)},
$$

is traceless for an incompressible fluid and does not show up in the equation for turbulent kinetic energy, which is found by taking $\frac{1}{2}$ the trace of Eq. (9). Therefore, this term simply redistributes energy from the streamwise velocity fluctuations to the wall-normal and spanwise velocity fluctuations. As stated earlier, there is no production of either $\overline{v_{y}^{\prime} v_{y}^{\prime}}$ or $\overline{v_{z}^{\prime} v_{z}^{\prime}}$ by the term of Eq. (12), so the terms of the pressure-rate-ofstrain act as a pseudoproduction term for wall-normal and spanwise velocity fluctuations. The pressure-rate-of-strain term [Eq. (13)] and the pressure transport term [Eq. (11)] are a decomposition of the velocity-pressure-gradient term, 


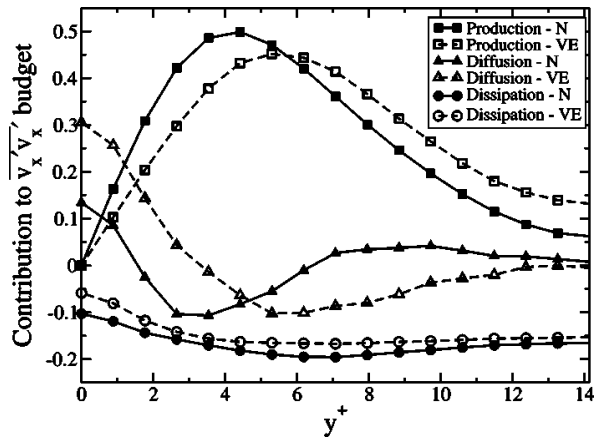

FIG. 15. The contribution to the $\overline{v_{x}^{\prime} v_{x}^{\prime}}$ budget of the production, diffusion, and dissipation terms for Newtonian $(\operatorname{Re}=200, \beta=1)$ and viscoelastic $(\operatorname{Re}$ $=200, \mathrm{We}=16.0, \mathrm{Ex}=100$, and $\beta=0.97$ ) solutions. Only half of the domain is shown — by symmetry all statistical quantities are symmetric across the Couette cell centerline $y^{+}=\sqrt{\operatorname{Re}}$.

$$
\mathrm{II}_{i j}=R_{i j}+T_{i j}^{p}=-\overline{v_{i}^{\prime} \frac{\partial p^{\prime}}{\partial x_{j}}+v_{j}^{\prime} \frac{\partial p^{\prime}}{\partial x_{i}}} .
$$

The diffusion and dissipation of Reynolds stresses are given by

$$
D_{i j}=\frac{\beta}{\operatorname{Re}} \frac{\partial^{2}}{\partial x_{k} \partial x_{k}} \overline{v_{i}^{\prime} v_{j}^{\prime}}
$$

and

$$
\varepsilon_{i j}=-\frac{2 \beta}{\operatorname{Re}} \overline{\frac{\partial v_{i}^{\prime}}{\partial x_{k}} \frac{\partial v_{j}^{\prime}}{\partial x_{k}},}
$$

respectively. The direct contribution of the polymer stresses to the Reynolds stress budgets is the velocity-polymer-force term

$$
E_{i j}=\overline{v_{i}^{\prime} f_{j}^{\prime}+v_{j}^{\prime} f_{i}^{\prime}} .
$$

Note that for the current exact coherent states, vorticity boundary conditions are used. This choice of boundary conditions eliminates the viscous sublayer. Therefore, when the wall is referenced in this section, it should be understood that this refers to the boundary of the buffer layer.

The contributions of the production, dissipation, and diffusion terms to the $\overline{v_{x}^{\prime} v_{x}^{\prime}}$ budget are compared for a Newtonian $(\mathrm{Re}=200)$ solution and a viscoelastic solution $(\mathrm{Re}=200$ and $\mathrm{We}=16$ ) in Fig. 15. For the viscoelastic flow, the production term decreases compared to the Newtonian near the wall. Due to the use of vorticity boundary conditions in this study, the $\partial \bar{V}_{x} / \partial y$ contribution to the production is the same for both the viscoelastic and Newtonian flow near the wall. Therefore, this reduction in $P_{x x}$ near the wall must be due to decreases in $v_{x}^{\prime}$ and $v_{y}^{\prime}$. Farther from the wall, differences between $\partial \bar{V}_{x} / \partial y$ in the viscoelastic and Newtonian flow is apparent as the production for the viscoelastic flow becomes greater than for the Newtonian. Since the polymer reduces the strength of the vortices and thus the pressure fluctuations, the redistribution of the mean shear is reduced and $\partial \bar{V}_{x} / \partial y$ increases near the centerline for the viscoelastic flow (Fig. $5)$. The increase in the production term in the $\overline{v_{x}^{\prime} v_{x}^{\prime}}$ budget away from the wall is one factor in the increase of stream-

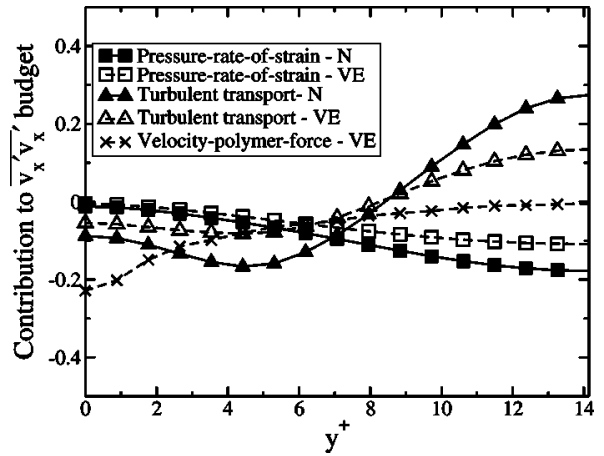

FIG. 16. The contribution to the $\overline{v_{x}^{\prime} v_{x}^{\prime}}$ budget of the pressure-rate-of-strain, turbulent transport, and velocity-polymer-force terms for Newtonian (Re $=200, \beta=1)$ and viscoelastic $(\operatorname{Re}=200, \mathrm{We}=0.16, \mathrm{Ex}=100$, and $\beta=0.97)$ solutions.

wise velocity fluctuations in this region. (The other factors increasing these fluctuations are discussed below.) These trends in the effects of the polymer on the production, diffusion, and dissipation terms in the ECS are qualitatively the same as found via DNS of drag-reducing fluids. ${ }^{18}$ Specific numbers are different because of the differences in geometry and parameters, but as an example of the relationship between our ECS results and those from DNS, Dimitropoulos et al. ${ }^{18}$ find in channel flow DNS that the maximum value of $P_{11}$ is 0.3 for $\mathrm{Re}_{\tau}=125, \mathrm{We}=50$, and $\mathrm{Ex} \approx 66$; for the viscoelastic Couette flow ECS at the conditions of Fig. 15 we find a maximum value of 0.45 .

Figure 16 shows the remaining contributions to the $\overline{v_{x}^{\prime} v_{x}^{\prime}}$ budget. The pressure-rate-of-strain term in this budget decreases in magnitude due to the addition of polymer. As stated above, the pressure-rate-of-strain term acts to transfer energy from the streamwise velocity fluctuations to the wallnormal and spanwise fluctuating velocities. Since this term is reduced in magnitude, the transfer of energy is reduced and the streamwise velocity fluctuations increase as seen in Fig. 6. The decrease in the pressure-rate-of-strain term is due to the reduction of the pressure fluctuations. The pressure fluctuations in the ECS deviate most from the mean in the cores of the vortices. The decrease of vortex strength due to the polymer causes the magnitude of the pressure fluctuations to decrease and, likewise, the redistribution due to the pressurerate-of-strain term. The direct contribution of the polymer to the $\overline{v_{x}^{\prime} v_{x}^{\prime}}$ budget is also seen in Fig. 16. Notice that this contribution is largest near the wall, indicating that the polymer stretch (and stress) is highest in the streamwise streaks, which matches the proposed mechanism. The direct contribution of the polymer approaches zero farther from the wall where the changes in the streamwise fluctuations are largest. This indicates that the effects of the polymer are more complicated than what is simply captured in the velocitypolymer-force term.

Figures 17 and 18 show the contributions to the $\overline{v_{x}^{\prime} v_{y}^{\prime}}$ budgets. The production term, which acts as a sink in this case, decreases in magnitude for the viscoelastic fluid. However, the pressure-rate-of-strain term for this budget, which acts to increase Reynolds shear stress by redistributing energy from the streamwise fluctuations, also decreases. This is 


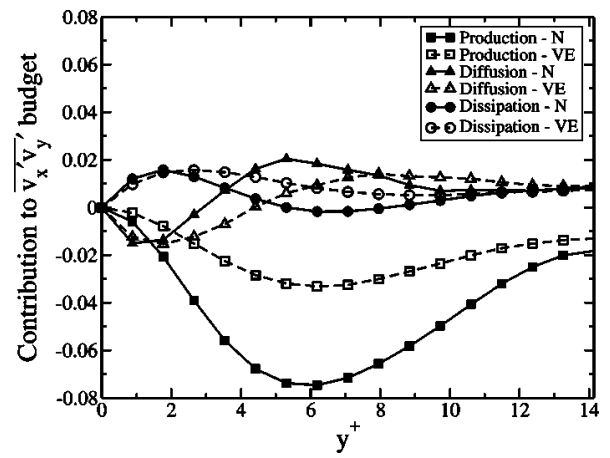

FIG. 17. The contribution to the $\overline{v_{x}^{\prime} v_{y}^{\prime}}$ budget of the production, diffusion, and dissipation terms for Newtonian $(\operatorname{Re}=200, \beta=1)$ and viscoelastic ( $\operatorname{Re}$ $=200, \mathrm{We}=0.16, \mathrm{Ex}=100$, and $\beta=0.97)$ solutions.

again due to the polymer weakening the vortices and reducing the pressure fluctuations. The reduction in the pressurerate-of-strain term (source) is greater than the increase in production term, especially near the center of the channel, and the net effect is a decrease in the Reynolds shear stress. The velocity-pressure gradient term (Fig. 19), which is the sum of the $R_{x y}$ and $T_{x y}^{p}$ terms, decreases for the viscoelastic ECS. The velocity-polymer-force term is small in this budget because the polymer are stretched primarily in the streamwise direction (so $f_{y}^{\prime}$ is small) and stretching primarily occurs near the wall (so $v_{y}^{\prime}$ is smallest where $f_{x}^{\prime}$ is largest). These results closely match those of Dimitropoulos et al. $^{18}$

Figures 20-22 show the contributions to the $\overline{v_{y}^{\prime} v_{y}^{\prime}}$ budget. Recall that for the $\overline{v_{y}^{\prime} v_{y}^{\prime}}$ budget, the production term is zero for shear turbulence and also for the ECS. However, the pressure-rate-of-strain term acts to transfer energy from the streamwise fluctuating velocity to the wall-normal velocity. Figure 21 shows that the pressure-rate-of-strain term decreases in magnitude in the viscoelastic case, reducing the transfer of energy between the velocity components. As mentioned previously, this change in the pressure-rate-of-strain term is caused when the polymer reduces the pressure fluctuations by weakening the streamwise vortices. The velocitypolymer-force term in this budget is small and negative, em-

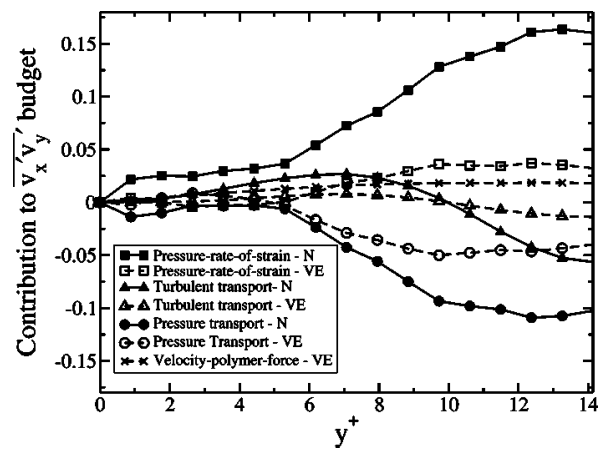

FIG. 18. The contribution to the $\overline{v_{x}^{\prime} v_{y}^{\prime}}$ budget of the pressure-rate-of-strain, turbulent transport, pressure transport, and velocity-polymer-force terms for Newtonian $(\operatorname{Re}=200, \beta=1)$ and viscoelastic $(\mathrm{Re}=200, \mathrm{We}=0.16, \mathrm{Ex}=100$, and $\beta=0.97$ ) solutions.

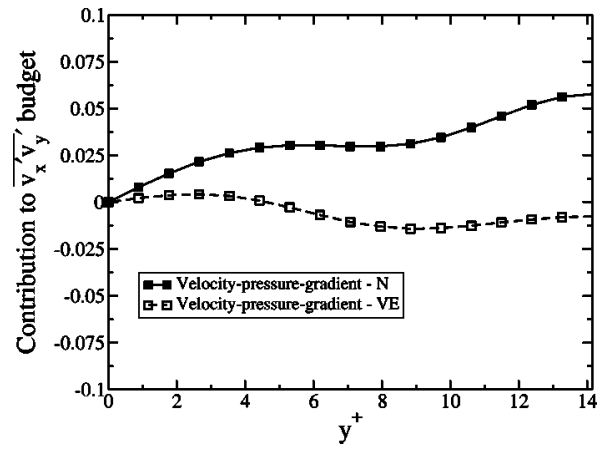

FIG. 19. The contribution to the $\overline{v_{x}^{\prime} v_{y}^{\prime}}$ budget of the velocity-pressuregradient term for Newtonian $(\operatorname{Re}=200, \beta=1)$ and viscoelastic $(\operatorname{Re}=200$, $\mathrm{We}=0.16, \mathrm{Ex}=100$, and $\beta=0.97)$ solutions.

phasizing again that the effects of viscoelasticity on the Reynolds stresses are not simply related to the direct contribution of the polymer force.

All of the results presented here qualitatively match those of the DNS study by Dimitropoulos et al. ${ }^{18}$ The effects of viscoelasticity on the production (and pseudo-production) terms can be attributed to the suppression of the vortices by the polymer. The effects of streamwise vortex suppression are twofold: First, the redistribution of mean shear due to the vortices is reduced, increasing the net production of streamwise velocity fluctuations by the mean shear. Second, the pressure fluctuations, which show the largest deviation from the mean pressure in the vortex cores, are reduced, decreasing the transfer of energy from the streamwise velocity fluctuations to the wall-normal and spanwise directions. The structural mechanism for drag reduction proposed in the previous section is consistent with the Reynolds stress results.

\section{CONCLUSIONS}

We have studied the effects of viscoelasticity on nontrivial, traveling-wave solutions in plane Couette flow that serve as a minimal model for the turbulent buffer region. These traveling-wave solutions, called "exact coherent states" (ECS), capture the dominant structure of the buffer region: pairs of streamwise-aligned vortices that lead to steaks in the streamwise velocity. The optimum length scales for the ECS in plane Poiseuille flow quantitatively match the

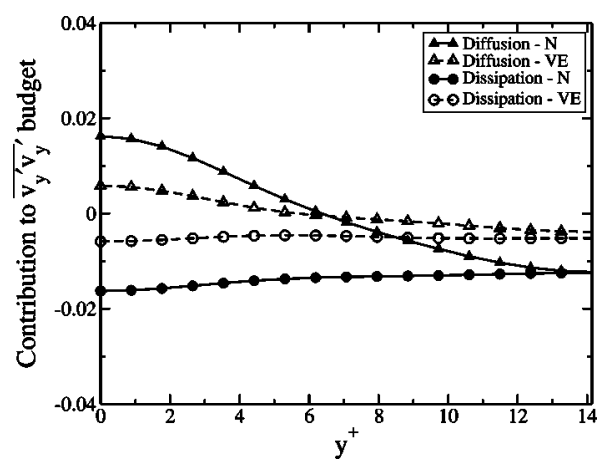

FIG. 20. The contribution to the $\overline{v_{y}^{\prime} v_{y}^{\prime}}$ budget of the diffusion and dissipation terms for Newtonian $(\operatorname{Re}=200, \beta=1)$ and viscoelastic $(\mathrm{Re}=200$, We $=0.16, \mathrm{Ex}=100$, and $\beta=0.97$ ) solutions. 


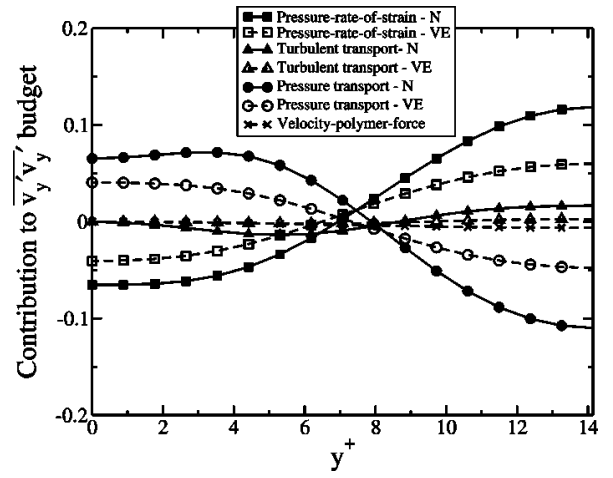

FIG. 21. The contribution to the $\overline{v_{y}^{\prime} v_{y}^{\prime}}$ budget of the pressure-rate-of-strain, turbulent transport, pressure transport, and velocity-polymer-force terms for Newtonian $(\operatorname{Re}=200, \beta=1)$ and viscoelastic $(\operatorname{Re}=200, \mathrm{We}=0.16, \mathrm{Ex}=100$, and $\beta=0.97$ ) solutions.

length scales of the turbulent coherent structures observed in experiments and recent experimental ${ }^{30}$ and DNS evidence ${ }^{35-37}$ suggests that the ECS underlie the dynamics of these structures. In the variant of Couette flow that we study here, above a certain Weissenberg number $\mathrm{We}_{\text {onset }} \approx 7$, there is dramatic increase in the minimum Reynolds number at which the ECS can exist. Since the minimum Reynolds number is related to the minimum wall-normal size for the existence of the ECS $\left[\left(\operatorname{Re}_{\min }=\frac{1}{4}\left(\Delta y_{\min }^{+}\right)^{2}\right)\right]$, one effect of viscoelasticity is to shift the mechanism that maintains the ECS to larger wall-normal length scales, mirroring experimental observations of buffer region "thickening" in drag reduced flows. Since evidence indicates that the existence of the ECS is a prerequisite for transition to turbulence, the increase in $\mathrm{Re}_{\min }$ is also consistent with the experimental observed delay in transition for polymer solutions.

The mechanism that is responsible for the upward shift of $\mathrm{Re}_{\min }$ is one of vortex suppression. For the viscoelastic ECS above $\mathrm{We}_{\text {onset, }}$, there is a rapid decrease in the rms, wall-normal, and spanwise velocity fluctuations and the rms, streamwise vorticity. Since the magnitudes $v_{y}^{\prime}$ and $v_{z}^{\prime}$ are greatest at the edges of the streamwise vortices, the decrease in their values, and that of $\omega_{x}^{\prime}$, indicate that the polymer is weakening the vortices. A result of the suppression of the vortices is a decrease in the magnitude of the pressure fluctuations in the vortex cores (i.e., they become less negative).

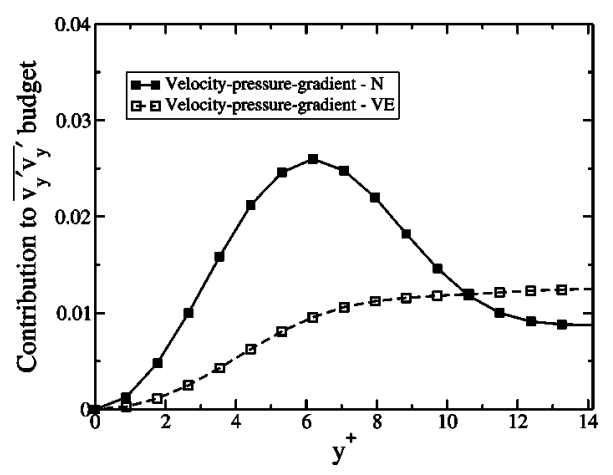

FIG. 22. The contribution to the $\overline{v_{y}^{\prime} v_{y}^{\prime}}$ budget of the velocity-pressuregradient term for Newtonian $(\operatorname{Re}=200, \beta=1)$ and viscoelastic $(\operatorname{Re}=200$, $\mathrm{We}=0.16, \mathrm{Ex}=100$, and $\beta=0.97)$ solutions.

\section{Vortex suppression by polymer}

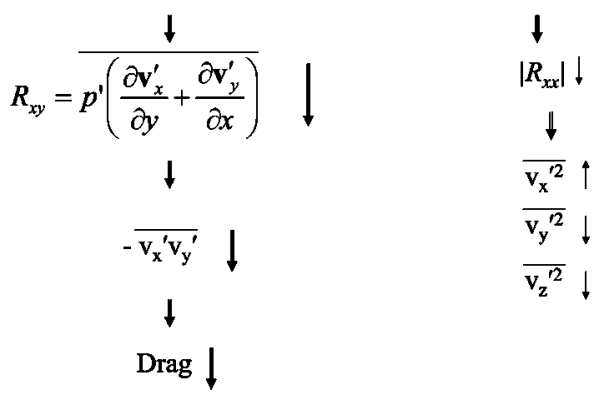

FIG. 23. Summary of the effects of the polymer on the Reynolds stresses.

The suppression of the vortices is understood as follows: The polymers become highly stretched moving through the streamwise velocity streak and relax as they move out of the streak, into and around a vortex; the polymer relaxation produces a force that directly opposes the motion of fluid elements entering the vortex, slowing them, and weakening the vortex. While this work has only presented results for the ECS in plane Couette flow, our preliminary results for viscoelastic ECS in plane Poiseuille flow show the same mechanism and qualitatively similar changes to the velocity fluctuations. $^{34}$

The proposed mechanism also explains the changes measured in the Reynolds shear stress, $v_{x}^{\prime} v_{y}^{\prime}$ and fluctuating velocities at low to moderate levels of drag reduction, as shown in Fig. 23. In the budget of Reynolds stresses, the pressure-rate-of-strain term acts to redistribute energy from the streamwise velocity fluctuations to the wall-normal and spanwise velocity fluctuations and the Reynolds shear stress. This redistribution term depends on the pressure fluctuations, which are of highest magnitude in the vortex cores. Since the polymer weakens the vortices, the magnitude of the pressure fluctuations decreases as We increases and, as a consequence, the pressure-rate-of-strain term decreases in magnitude. Transfer of energy from the streamwise fluctuations to the Reynolds shear stress diminishes. The reduction in the Reynolds shear stress leads to drag reduction.

The viscoelastic ECS comprise a minimal description of near-wall turbulence in a polymer solutions. They are autonomous, and the effects of polymer on these states mirror DNS and experimental observations near the onset of drag reduction: structures shift to larger wall-normal length scales, streamwise velocity fluctuations are enhanced while wallnormal and spanwise fluctuations, streamwise vorticity fluctuations and Reynolds shear stress decrease, and drag is reduced.

At high levels of drag reduction, the character of drag reduced flow changes significantly. The log-layer mean velocity gradient (inverse von Kármán constant) is significantly higher than the Newtonian value over a large fraction of the channel. ${ }^{1}$ Furthermore, as the level of drag reduction increases, the streamwise velocity fluctuations (scaled with $u_{\tau}$ ) peak and then decrease, ${ }^{51,52}$ a result that suggests that at high drag reduction a change in the basic turbulence production 
mechanism occurs. The relevance of the viscoelastic ECS in this regime is not clear.

\section{ACKNOWLEDGMENTS}

The authors gratefully acknowledge support from NSF and the donors of the Petroleum Research Fund, administered by the American Chemical Society.

${ }^{1}$ P. S. Virk, "Drag reduction fundamentals," AIChE J. 21, 225 (1975).

${ }^{2}$ J. L. Lumley, "Drag reduction by additives," Annu. Rev. Fluid Mech. 1, 367 (1969).

${ }^{3}$ W. D. McComb, The Physics of Fluid Turbulence (Oxford University Press, New York, 1990).

${ }^{4}$ M. D. Graham, "Drag reduction in turbulent flow of polymer solutions," in Rheology Reviews 2004 (British Society of Rheology, 2004).

${ }^{5}$ G. L. Donohue, W. G. Tiederman, and M. M. Reischman, "Flow visualization of the near-wall region in a drag-reducing channel flow," J. Fluid Mech. 50, 559 (1972).

${ }^{6}$ W. G. Tiederman, T. S. Luchik, and D. G. Bogard, "Wall-layer structure and drag reduction," J. Fluid Mech. 156, 419 (1985).

${ }^{7}$ D. T. Walker and W. G. Tiederman, "Turbulent structure in a channel flow with polymer injection at the wall," J. Fluid Mech. 218, 377 (1990).

${ }^{8}$ M. P. Escudier, F. Presti, and S. Smith, "Drag reduction in the turbulent pipe flow of polymers," J. Non-Newtonian Fluid Mech. 81, 197 (1999).

${ }^{9}$ S. K. Robinson, "Coherent motions in the turbulent boundary layer," Annu. Rev. Fluid Mech. 23, 601 (1991).

${ }^{10}$ P. Holmes, J. L. Lumley, and G. Berkooz, Turbulence, Coherent Structures, Dynamical Systems and Symmetry (Cambridge University Press, New York, 1996).

${ }^{11}$ S. B. Pope, Turbulent Flows (Cambridge University Press, New York, 2000).

${ }^{12}$ R. Sureshkumar, A. N. Beris, and R. Handler, "Direct numerical simulation of the turbulent channel flow of a polymer solution," Phys. Fluids 9, 743 (1997)

${ }^{13}$ J. M. J. den Toonder, M. A. Hulsen, G. D. C. Kuiken, and F. T. M. Nieuwstadt, "Drag reduction by polymer additives in a turbulent pipe flow: numerical and laboratory experiments," J. Fluid Mech. 337, 193 (1997).

${ }^{14}$ A. A. Draad, G. D. C. Kuiken, and F. T. M. Nieuwstadt, "Laminarturbulent transition in pipe flow for Newtonian and Non-Newtonian fluids,” J. Fluid Mech. 377, 267 (1998).

${ }^{15}$ J. L. Lumley, "Drag reduction in turbulent flow by polymer additives," J. Polym. Sci., Macromol. Rev. 7, 263 (1973).

${ }^{16} \mathrm{~T}$. Wei and W. W. Willmarth, "Modifying turbulent structure with dragreducing polymer additives in turbulent channel flows," J. Fluid Mech. 245, 619 (1992)

${ }^{17}$ P. K. Ptasinski, B. J. Boersma, F. T. M. Nieuwstadt, M. A. Hulsen, B. H. A. A. V. D. Brule, and J. C. R. Hunt, "Turbulent channel flow near maximum drag reduction: simulations, experiments and mechanisms," J. Fluid Mech. 490, 251 (2003).

${ }^{18}$ C. D. Dimitropoulos, R. Sureshkumar, A. N. Beris, and R. A. Handler, "Budgets of Reynolds stress, kinetic energy and streamwise enstrophy in viscoelastic turbulent channel flow," Phys. Fluids 13, 1016 (2001).

${ }^{19}$ K. D. Housiadas and A. N. Beris, "Polymer-induced drag reduction: Effects of variations in elasticity and inertia in turbulent viscoelastic channel flow," Phys. Fluids 15, 2369 (2003).

${ }^{20}$ M. Nagata, "Bifurcation in Couette flow between almost corotating cylinders," J. Fluid Mech. 169, 229 (1986).

${ }^{21}$ M. Nagata, "On wavy instabilities of the Taylor-vortex flow between corotating cylinders," J. Fluid Mech. 188, 585 (1988).

${ }^{22}$ M. Nagata, "Three-dimensional finite amplitude solutions in plane Couette flow: bifurcation from infinity," J. Fluid Mech. 217, 519 (1990).

${ }^{23}$ R. M. Clever and F. H. Busse, "Tertiary and quaternary solutions for plane Couette flow," J. Fluid Mech. 344, 137 (1997).

${ }^{24} \mathrm{~F}$. Waleffe, "Three-dimensional coherent states in plane shear flows," Phys. Rev. Lett. 81, 4140 (1998).
${ }^{25}$ F. Waleffe, "Exact coherent structures in channel flow," J. Fluid Mech. 435, 93 (2001).

${ }^{26} \mathrm{~F}$. Waleffe, "Homotopy of exact coherent structures in plane shear flows," Phys. Fluids 15, 1517 (2003).

${ }^{27}$ J. Jeong, F. Hussian, W. Schoppa, and J. Kim, "Coherent structures near the wall in a turbulent channel flow," J. Fluid Mech. 332, 185 (1997).

${ }^{28}$ H. Faisst and B. Eckhardt, "Traveling waves in pipe flow," Phys. Rev. Lett. 90, 224502 (2003).

${ }^{29}$ S. H. Strogatz, Nonlinear Dynamics and Chaos: With Applications to Physics, Biology, Chemistry and Engineering (Addison-Wesley, New York, 1994).

${ }^{30}$ S. Bottin, O. Dauchot, F. Daviaud, and P. Mannveille, "Experimental evidence of streamwise vorticies as finite amplitude solutions in transitional plane Couette flow," Phys. Fluids 10, 2597 (1998).

${ }^{31}$ D. R. Carlson, S. E. Widnall, and M. F. Peeters, "A flow-visualization study of transition in plane Poiseuille flow," J. Fluid Mech. 121, 487 (1982).

${ }^{32}$ R. B. Bird, W. E. Stewart, and E. N. Lightfoot, Transport Phenomena, 2nd ed. (Wiley, New York, 2002).

${ }^{33}$ J. Jiménez and P. Moin, "The minimal flow unit in near wall turbulence," J. Fluid Mech. 225, 221 (1991).

${ }^{34}$ P. A. Stone, Ph.D. thesis, University of Wisconsin-Madison, 2004.

${ }^{35}$ G. Kawahara and S. Kida, "Periodic motion embedded in plane Couette turbulence: regeneration cycle and burst," J. Fluid Mech. 449, 291 (2001).

${ }^{36} \mathrm{~S}$. Toh and T. Itano, "A periodic-like solution in channel flow," J. Fluid Mech. 481, 67 (2003).

${ }^{37}$ J. Jiménez and M. P. Simens, "Low-dimensional dynamics of a turbulent wall flow," J. Fluid Mech. 435, 81 (2001).

${ }^{38}$ J. M. Hamilton, J. Kim, and F. Waleffe, "Regeneration mechanisms of near-wall turbulent structures," J. Fluid Mech. 287, 317 (1995).

${ }^{39} \mathrm{~F}$. Waleffe, "On a self-sustaining process in shear flows," Phys. Fluids $\mathbf{9}$, 883 (1997).

${ }^{40}$ P. G. Drazin and W. H. Reid, Hydrodynamic Stability (Cambridge University Press, New York, 1981).

${ }^{41}$ P. A. Stone, F. Waleffe, and M. D. Graham, "Toward a structural understanding of turbulent drag reduction: nonlinear coherent states in viscoelastic shear flows," Phys. Rev. Lett. 89, 208301 (2002).

${ }^{42}$ T. Min, J. Y. Yoo, H. Choi, and D. D. Joseph, "Drag reduction by polymer additives in a turbulent channel flow," J. Fluid Mech. 486, 213 (2003).

${ }^{43}$ R. B. Bird, C. F. Curtiss, R. C. Armstrong, and O. Hassager, Dynamics of Polymeric Liquids, 2nd ed. (Wiley, New York, 1987), Vol. 2.

${ }^{44}$ P. A. Stone and M. D. Graham, "Polymer dynamics in a model of the turbulent buffer layer," Phys. Fluids 15, 1247 (2003).

${ }^{45}$ T. Min, J. Y. Yoo, and H. Choi, "Effect of spatial discretization schemes on numerical solution of viscoelastic fluid flows," J. Non-Newtonian Fluid Mech. 100, 27 (2001).

${ }^{46}$ Y. Dubief and S. K. Lele, "Direct numerical simulation of polymer flow," in Center for Turbulence Research Annual Research Briefs (Stanford University, 2001), pp. 197-204.

${ }^{47}$ Y. Dubief, V. E. Terrapon, C. M. White, E. Shaqfeh, P. Moin, and S. Lele, "New answers on the interaction between polymers and vortices in turbulent flows," Flow, Turbul. Combust. (to be published).

${ }^{48}$ R. Sureshkumar and A. N. Beris, "Effect of artificial stress diffusivity on the stability of numerical calculations and the flow dynamics of timedependent viscoelastic flows," J. Non-Newtonian Fluid Mech. 60, 53 (1995).

${ }^{49} \mathrm{~S}$. Sibilla and A. Baron, "Polymer stress statistics in the near-wall turbulent flow of a drag-reducing solution," Phys. Fluids 14, 1123 (2002).

${ }^{50}$ E. De Angelis, C. M. Casciola, and R. Piva, "DNS of wall turbulence: dilute polymers and self-sustaining mechanisms," Comput. Fluids 31, 495 (2002).

${ }^{51}$ P. K. Ptasinski, F. T. M. Nieuwstadt, B. H. A. A. van den Brule, and M. A. Hulsen, "Experiments in turbulent pipe flow with polymer additives at maximum drag reduction," Flow, Turbul. Combust. 66, 159 (2001).

${ }^{52}$ M. D. Warholic, H. Massah, and T. J. Hanratty, "Influence of dragreducing polymers on turbulence: Effects of Reynolds number, concentration and mixing," Exp. Fluids 27, 461 (1999). 